\title{
A New Bicyclic Dipeptide Isostere with Pyrrolizidinone Skeleton
}

\author{
F. M. Cordero, F. Pisaneschi, K. Meschini Batista, S. Valenza, F. Machetti, A. Brandi
}

Dipartimento di Chimica Organica "Ugo Schiff”, Università degli Studi di Firenze, and ICCOM-

CNR, via della Lastruccia 13, I-50019 Sesto Fiorentino (Fi), Italy.

franca.cordero@unifi.it

\section{SUPPORTING INFORMATION}

\section{Contents List:}

General Experimental Methods

Conformational Analysis, NMR and IR Methods

X-ray Structural Analyses of exo-6 and (2R,7aR)-23a

Compound exo-6

Crystal data and structure refinement

Atomic coordinates and equivalent isotropic displacement parameters

Bond lengths and angles

Anisotropic displacement parameters

Compound $(2 R, 7 \mathrm{a} R)-\mathbf{2 3 a}$

Crystal data and structure refinement

Atomic coordinates and equivalent isotropic displacement parameters

Bond lengths and angles

Anisotropic displacement parameters

References
Page

S2

S2

S2

S3

S3

S4

S5

S6

S7

S7

S8

S8

S11

S11 
General Experimental Methods: All the reactions requiring anhydrous conditions were carried out under nitrogen and the solvents were appropriately dried before use. NMR spectra were recorded in $\mathrm{CDCl}_{3}$ (except where indicated) and the data are reported in $\delta(\mathrm{ppm})$ from TMS. The temperature dependence $\Delta \delta / \Delta \mathrm{T}$ was measured by collecting data from 295 to $320 \mathrm{~K}$ in steps of $5 \mathrm{~K}$ using a variable temperature probe at $400 \mathrm{MHz}$. Multiplicity of the ${ }^{13} \mathrm{C}$ NMR was determined by means of APT and HMQC experiments. In mass spectra relative percentages are shown in brackets. $R_{\mathrm{f}}$ values refer to TLC on $0.25 \mathrm{~mm}$ silica gel plates and were measured (except where indicated) using the same eluant employed in the purification of the corresponding compounds.

Conformational Analysis, NMR and IR Methods: All NMR experiments were performed on a $400 \mathrm{MHz}$ spectrometer, using $2 \mathrm{mM}$ solutions of pseudotripeptides $\mathbf{1 8}, \mathbf{2 1}$ and 22 in $\mathrm{CDCl}_{3}(800$ $\mu \mathrm{L})$. Variable-temperature NMR (VT NMR) measurements were performed in a temperature range of 295-320 K. DMSO titration was carried out adding tenfold $1 \%$ amount of DMSO-d ${ }_{6}(8 \mu \mathrm{L} \times 10$ times) to the $\mathrm{CDCl}_{3}$ solution in the NMR tube and recording a spectrum after each addition. IR spectra were recorded on a FT-IR system, using $30 \mathrm{mM}$ and $7 \mathrm{mM}$ solutions of 18, 21 and 22 in $\mathrm{CH}_{2} \mathrm{Cl}_{2}$ at $\mathrm{rt}$; in each case the spectrum of the solvent was subtracted from that of the solution under investigation. 


\section{$\mathrm{X}$-ray Structural Analyses of exo-6 and $(2 R, 7 a R)-23 a$}

Analyses on single crystals were carried out at rt. Graphite-monochromated $\mathrm{Cu} \mathrm{K} \alpha$ radiation was used for cell parameter determinations and data collection. The intensities of two standard reflections were monitored during data collections to check the stability of the crystals: no loss of intensities was recognized. The integrated intensities, measured using the $\theta / 2 \theta$ scan mode, were corrected for Lorentz and polarization effects. ${ }^{\mathrm{S} 1}$ The non-hydrogen atoms were refined anisotropically, whereas hydrogen atoms were refined as isotropic. The structures were solved by direct methods of SIR97 ${ }^{\mathrm{S} 2}$ and refined using the full-matrix least squares on $\mathrm{F}^{2}$ provided by SHELXL97. ${ }^{\text {S3 }}$

Aromatic and methyl hydrogens were assigned in calculated positions, the others were found in the Fourier difference synthesis.

Compound exo-6: $\mathrm{C}_{9} \mathrm{H}_{14} \mathrm{~N}_{2} \mathrm{O}_{4}, \mathrm{M}=214.22$, Triclinic, space group $\mathrm{P}-1, a=5.178(1), b=7.536(1)$, $c=14.232(1) \AA, \alpha=95.170(9), \beta=99.840(10), \gamma=108.990(10) \operatorname{deg}, V=511.03(13) \AA^{3}, Z=2 D_{c}=1.392$, $\mu=0.932 \mathrm{~mm}^{-1}, \mathrm{~F}(000)=228$. The reflections collected were 2072 with a $3.19<\theta<59.97$ range; 1498 were independent and the final $\mathrm{R}$ index was 0.0484 for reflections having $\mathrm{I}>2 \sigma \mathrm{I}$, and 0.0503 for all data.

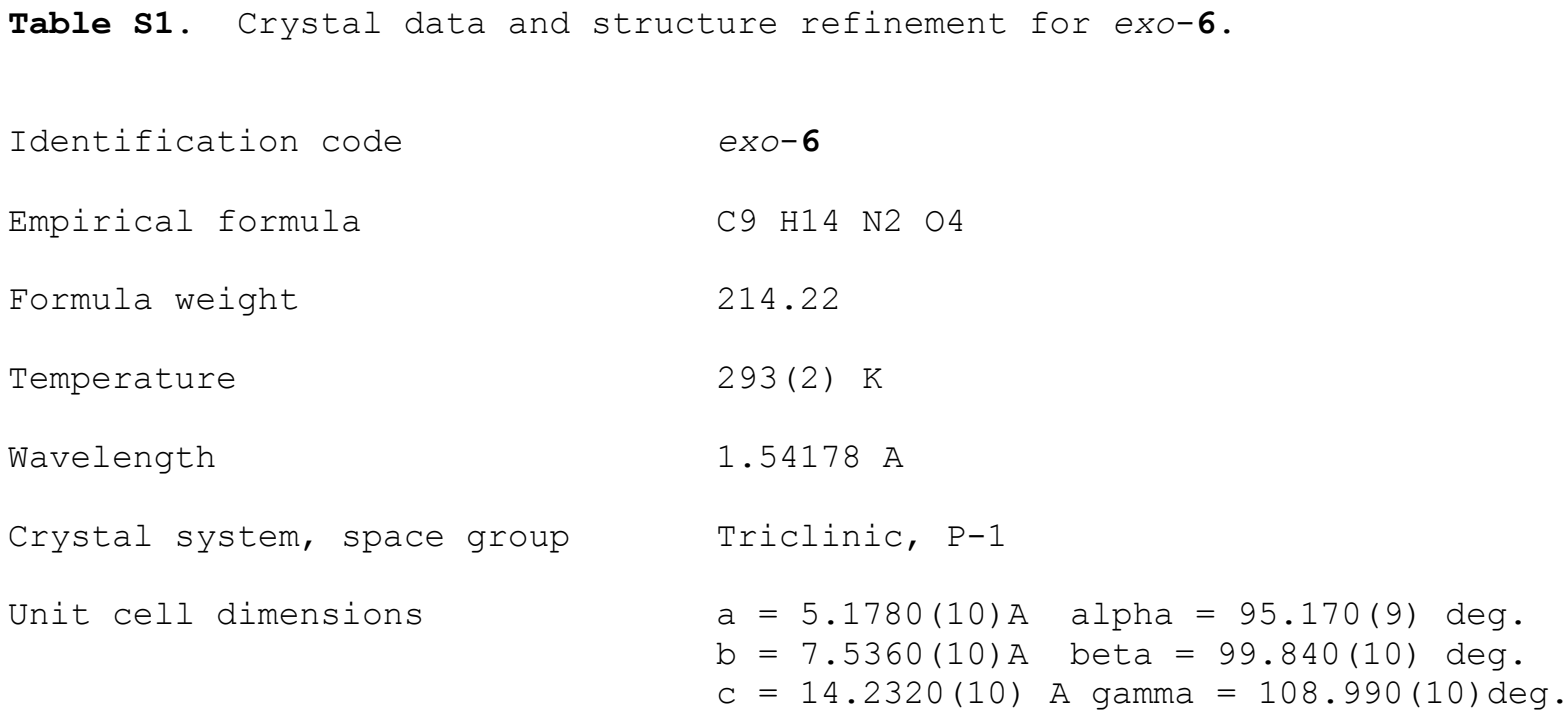




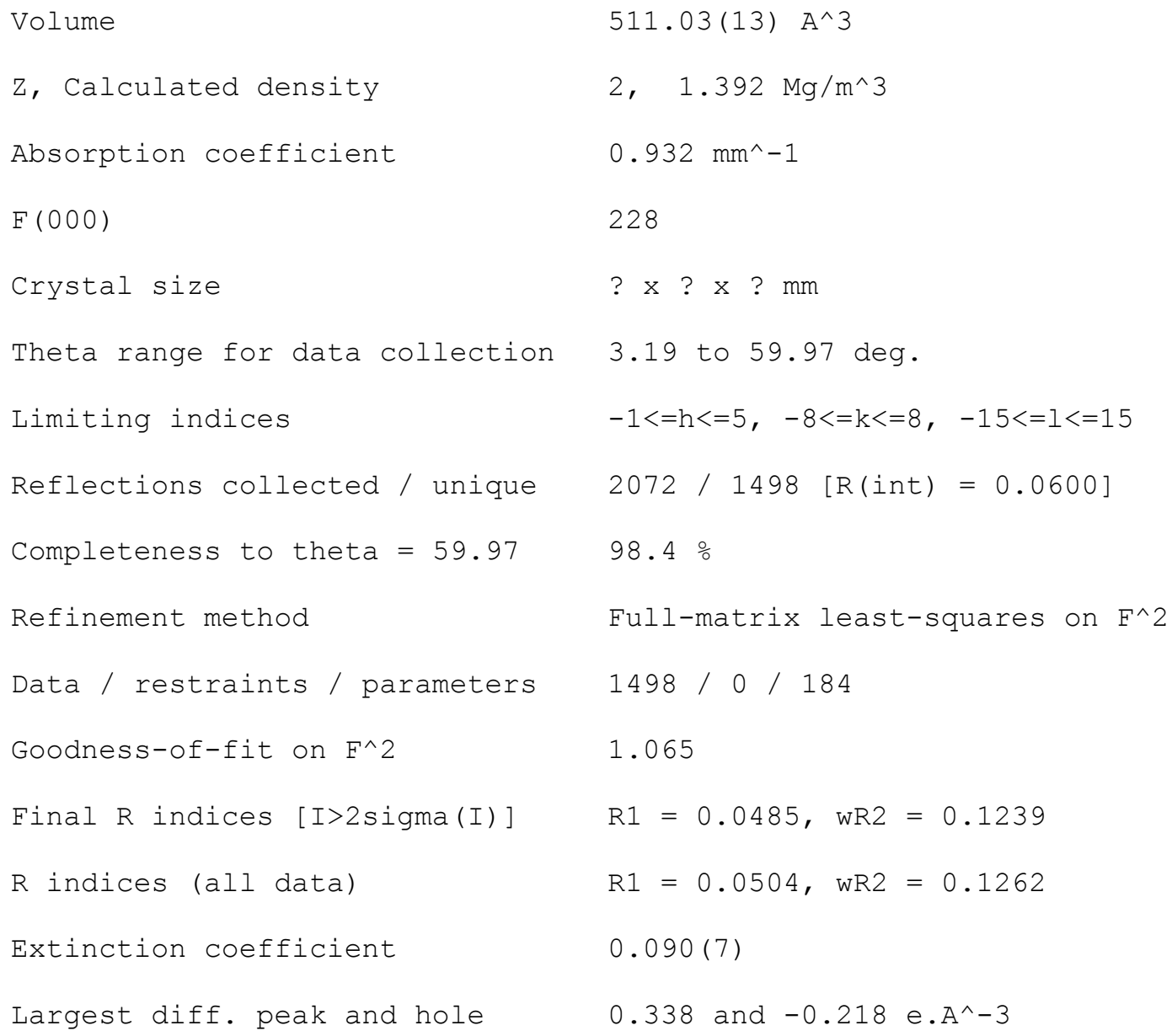

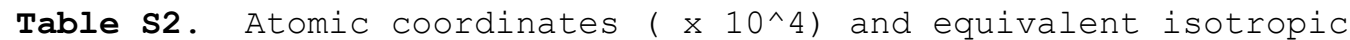
displacement parameters $\left(A^{\wedge} 2 \times 10^{\wedge} 3\right)$ for exo-6.

$\mathrm{U}(\mathrm{eq})$ is defined as one third of the trace of the orthogonalized Uij tensor.

\begin{tabular}{|c|c|c|c|c|}
\hline & $\mathrm{x}$ & $\mathrm{Y}$ & $\mathrm{z}$ & $\mathrm{U}(\mathrm{eq})$ \\
\hline$O(1)$ & $4043(3)$ & $-258(2)$ & 3702 (1) & $38(1)$ \\
\hline$O(2)$ & $7795(3)$ & $-3135(2)$ & $4650(1)$ & $47(1)$ \\
\hline$O(3)$ & $2682(5)$ & $-3557(3)$ & $1449(2)$ & $97(1)$ \\
\hline$O(4)$ & $1264(4)$ & $-1259(3)$ & $999(2)$ & $72(1)$ \\
\hline $\mathrm{N}(1)$ & $4112(4)$ & $673(2)$ & $2860(1)$ & $37(1)$ \\
\hline$N(2)$ & $3212(4)$ & $-3521(3)$ & $4377(1)$ & $42(1)$ \\
\hline$C(1)$ & $5367(4)$ & $-328(3)$ & $2218(2)$ & $38(1)$ \\
\hline$C(2)$ & $7018(6)$ & $1245(4)$ & $1699(2)$ & $53(1)$ \\
\hline$C(3)$ & $6701(8)$ & $3084(4)$ & $2140(3)$ & $76(1)$ \\
\hline$C(4)$ & $6037(6)$ & $2665(3)$ & 3101 (2) & $54(1)$ \\
\hline$C(5)$ & $6469(4)$ & $-823(3)$ & $3877(2)$ & $36(1)$ \\
\hline$C(6)$ & $5828(4)$ & $-2595(3)$ & $4345(1)$ & $35(1)$ \\
\hline$C(7)$ & $7158(6)$ & $-1169(4)$ & $2892(2)$ & $51(1)$ \\
\hline$C(8)$ & $2993(5)$ & $-1904(3)$ & $1523(2)$ & $46(1)$ \\
\hline C (9) & $-1088(7)$ & $-2630(5)$ & $318(2)$ & $79(1)$ \\
\hline
\end{tabular}


Table s3. Bond lengths [A] and angles [deg] for exo-6.

\begin{tabular}{|c|c|}
\hline $\mathrm{O}(1)-\mathrm{N}(1)$ & $1.442(2)$ \\
\hline$O(1)-C(5)$ & $1.443(2)$ \\
\hline $\mathrm{O}(2)-\mathrm{C}(6)$ & $1.242(2)$ \\
\hline $\mathrm{O}(3)-\mathrm{C}(8)$ & $1.195(3)$ \\
\hline $\mathrm{O}(4)-\mathrm{C}(8)$ & $1.310(3)$ \\
\hline$O(4)-C(9)$ & $1.444(3)$ \\
\hline$N(1)-C(4)$ & $1.477(3)$ \\
\hline$N(1)-C(1)$ & $1.492(3)$ \\
\hline$N(2)-C(6)$ & $1.317(3)$ \\
\hline $\mathrm{N}(2)-\mathrm{HN} 2 \mathrm{~A}$ & $0.81(3)$ \\
\hline $\mathrm{N}(2)-\mathrm{HN} 2 \mathrm{~B}$ & $0.93(4)$ \\
\hline$C(1)-C(8)$ & $1.524(3)$ \\
\hline$C(1)-C(7)$ & $1.539(3)$ \\
\hline$C(1)-C(2)$ & $1.548(3)$ \\
\hline$C(2)-C(3)$ & $1.539(4)$ \\
\hline $\mathrm{C}(2)-\mathrm{HC} 2 \mathrm{~A}$ & $0.94(3)$ \\
\hline $\mathrm{C}(2)-\mathrm{HC} 2 \mathrm{~B}$ & $1.03(4)$ \\
\hline$C(3)-C(4)$ & $1.502(4)$ \\
\hline $\mathrm{C}(3)-\mathrm{H}(3 \mathrm{~A})$ & $1.14(3)$ \\
\hline$C(3)-H(3 B)$ & $1.08(4)$ \\
\hline $\mathrm{C}(4)-\mathrm{H}(4 \mathrm{~A})$ & $0.91(4)$ \\
\hline $\mathrm{C}(4)-\mathrm{H}(4 \mathrm{~B})$ & $1.03(3)$ \\
\hline$C(5)-C(6)$ & $1.511(3)$ \\
\hline$C(5)-C(7)$ & $1.526(3)$ \\
\hline $\mathrm{C}(5)-\mathrm{H}(5)$ & $0.92(3)$ \\
\hline$C(7)-H(7 A)$ & $1.03(3)$ \\
\hline $\mathrm{C}(7)-\mathrm{H}(7 \mathrm{~B})$ & $0.96(3)$ \\
\hline $\mathrm{C}(9)-\mathrm{H}(9 \mathrm{~A})$ & 0.9600 \\
\hline$C(9)-H(9 B)$ & 0.9600 \\
\hline $\mathrm{C}(9)-\mathrm{H}(9 \mathrm{C})$ & 0.9600 \\
\hline $\mathrm{N}(1)-\mathrm{O}(1)-\mathrm{C}(5)$ & $107.96(13)$ \\
\hline$C(8)-O(4)-C(9)$ & $117.4(2)$ \\
\hline $\mathrm{O}(1)-\mathrm{N}(1)-\mathrm{C}(4)$ & $111.41(16)$ \\
\hline $\mathrm{O}(1)-\mathrm{N}(1)-\mathrm{C}(1)$ & $105.19(14)$ \\
\hline $\mathrm{C}(4)-\mathrm{N}(1)-\mathrm{C}(1)$ & $105.95(18)$ \\
\hline $\mathrm{C}(6)-\mathrm{N}(2)-\mathrm{HN} 2 \mathrm{~A}$ & $119.6(19)$ \\
\hline $\mathrm{C}(6)-\mathrm{N}(2)-\mathrm{HN} 2 \mathrm{~B}$ & $118.5(19)$ \\
\hline $\mathrm{HN} 2 \mathrm{~A}-\mathrm{N}(2)-\mathrm{HN} 2 \mathrm{~B}$ & $121(3)$ \\
\hline$N(1)-C(1)-C(8)$ & $108.02(16)$ \\
\hline $\mathrm{N}(1)-\mathrm{C}(1)-\mathrm{C}(7)$ & $105.58(17)$ \\
\hline$C(8)-C(1)-C(7)$ & $110.63(19)$ \\
\hline $\mathrm{N}(1)-\mathrm{C}(1)-\mathrm{C}(2)$ & $103.98(17)$ \\
\hline$C(8)-C(1)-C(2)$ & $112.65(19)$ \\
\hline$C(7)-C(1)-C(2)$ & $115.3(2)$ \\
\hline$C(3)-C(2)-C(1)$ & $105.4(2)$ \\
\hline $\mathrm{C}(3)-\mathrm{C}(2)-\mathrm{HC} 2 \mathrm{~A}$ & $116(2)$ \\
\hline $\mathrm{C}(1)-\mathrm{C}(2)-\mathrm{HC} 2 \mathrm{~A}$ & $110(2)$ \\
\hline $\mathrm{C}(3)-\mathrm{C}(2)-\mathrm{HC} 2 \mathrm{~B}$ & $110(2)$ \\
\hline $\mathrm{C}(1)-\mathrm{C}(2)-\mathrm{HC} 2 \mathrm{~B}$ & $108(2)$ \\
\hline $\mathrm{HC} 2 \mathrm{~A}-\mathrm{C}(2)-\mathrm{HC} 2 \mathrm{~B}$ & $108(3)$ \\
\hline$C(4)-C(3)-C(2)$ & $103.4(2)$ \\
\hline $\mathrm{C}(4)-\mathrm{C}(3)-\mathrm{H}(3 \mathrm{~A})$ & $72.7(14)$ \\
\hline$C(2)-C(3)-H(3 A)$ & $66.6(14)$ \\
\hline$C(4)-C(3)-H(3 B)$ & $111(2)$ \\
\hline $\mathrm{C}(2)-\mathrm{C}(3)-\mathrm{H}(3 \mathrm{~B})$ & $113(2)$ \\
\hline $\mathrm{H}(3 \mathrm{~A})-\mathrm{C}(3)-\mathrm{H}(3 \mathrm{~B})$ & $72(2)$ \\
\hline$N(1)-C(4)-C(3)$ & $102.2(2)$ \\
\hline$N(1)-C(4)-H(4 A)$ & $107(2)$ \\
\hline
\end{tabular}




$\begin{array}{ll}\mathrm{C}(3)-\mathrm{C}(4)-\mathrm{H}(4 \mathrm{~A}) & 117(2) \\ \mathrm{H}(3 \mathrm{~A})-\mathrm{C}(4)-\mathrm{H}(4 \mathrm{~A}) & 145(3) \\ \mathrm{N}(1)-\mathrm{C}(4)-\mathrm{H}(4 \mathrm{~B}) & 110.2(17) \\ \mathrm{C}(3)-\mathrm{C}(4)-\mathrm{H}(4 \mathrm{~B}) & 111.6(18) \\ \mathrm{H}(3 \mathrm{~A})-\mathrm{C}(4)-\mathrm{H}(4 \mathrm{~B}) & 70.5(19) \\ \mathrm{H}(4 \mathrm{~A})-\mathrm{C}(4)-\mathrm{H}(4 \mathrm{~B}) & 109(3) \\ \mathrm{O}(1)-\mathrm{C}(5)-\mathrm{C}(6) & 109.97(16) \\ \mathrm{O}(1)-\mathrm{C}(5)-\mathrm{C}(7) & 105.90(16) \\ \mathrm{C}(6)-\mathrm{C}(5)-\mathrm{C}(7) & 111.62(18) \\ \mathrm{O}(1)-\mathrm{C}(5)-\mathrm{H}(5) & 109.1(14) \\ \mathrm{C}(6)-\mathrm{C}(5)-\mathrm{H}(5) & 107.7(15) \\ \mathrm{C}(7)-\mathrm{C}(5)-\mathrm{H}(5) & 112.5(15) \\ \mathrm{O}(2)-\mathrm{C}(6)-\mathrm{N}(2) & 123.5(2) \\ \mathrm{O}(2)-\mathrm{C}(6)-\mathrm{C}(5) & 117.90(18) \\ \mathrm{N}(2)-\mathrm{C}(6)-\mathrm{C}(5) & 118.54(18) \\ \mathrm{C}(5)-\mathrm{C}(7)-\mathrm{C}(1) & 104.57(17) \\ \mathrm{C}(5)-\mathrm{C}(7)-\mathrm{H}(7 \mathrm{~A}) & 110.5(18) \\ \mathrm{C}(1)-\mathrm{C}(7)-\mathrm{H}(7 \mathrm{~A}) & 110.6(18) \\ \mathrm{C}(5)-\mathrm{C}(7)-\mathrm{H}(7 \mathrm{~B}) & 112.3(18) \\ \mathrm{C}(1)-\mathrm{C}(7)-\mathrm{H}(7 \mathrm{~B}) & 115.0(18) \\ \mathrm{H}(7 \mathrm{~A})-\mathrm{C}(7)-\mathrm{H}(7 \mathrm{~B}) & 104(3) \\ \mathrm{O}(3)-\mathrm{C}(8)-\mathrm{O}(4) & 122.3(2) \\ \mathrm{O}(3)-\mathrm{C}(8)-\mathrm{C}(1) & 125.2(2) \\ \mathrm{O}(4)-\mathrm{C}(8)-\mathrm{C}(1) & 112.53(18) \\ \text { O (4)-C (9)-H (9A) } & 109.5 \\ \text { O (4)-C (9)-H (9B) } & 109.5 \\ \mathrm{H}(9 \mathrm{~A})-\mathrm{C}(9)-\mathrm{H}(9 \mathrm{~B}) & 109.5 \\ \text { O (4)-C (9)-H (9C) } & 109.5 \\ \mathrm{H}(9 \mathrm{~A})-\mathrm{C}(9)-\mathrm{H}(9 \mathrm{C}) & 109.5 \\ \mathrm{H}(9 \mathrm{~B})-\mathrm{C}(9)-\mathrm{H}(9 \mathrm{C}) & 109.5 \\ & \end{array}$

Table s4. Anisotropic displacement parameters ( $A^{\wedge} 2 \times 10^{\wedge} 3$ ) for exo-6. The anisotropic displacement factor exponent takes the form: $-2 \mathrm{pi}^{\wedge} 2\left[\mathrm{~h}^{\wedge} 2 \mathrm{a}^{\star \wedge} 2 \mathrm{U} 11+\ldots+2 \mathrm{~h} k \mathrm{a}^{*} \mathrm{~b}\right.$ * $\mathrm{U} 12$ ]

\begin{tabular}{|c|c|c|c|c|c|c|}
\hline & U11 & $\mathrm{U} 22$ & U33 & $\mathrm{U} 23$ & U13 & U12 \\
\hline$O(1)$ & $38(1)$ & 41 (1) & $42(1)$ & $12(1)$ & $9(1)$ & $21(1)$ \\
\hline$O(2)$ & $34(1)$ & $45(1)$ & $70(1)$ & 22 (1) & $10(1)$ & $20(1)$ \\
\hline$O(3)$ & $104(2)$ & $43(1)$ & $125(2)$ & $1(1)$ & $-18(2)$ & $23(1)$ \\
\hline$O(4)$ & $73(1)$ & $56(1)$ & 72 (1) & $-12(1)$ & $-26(1)$ & $28(1)$ \\
\hline $\mathrm{N}(1)$ & $39(1)$ & $36(1)$ & $39(1)$ & $10(1)$ & $7(1)$ & $18(1)$ \\
\hline $\mathrm{N}(2)$ & $32(1)$ & $41(1)$ & $57(1)$ & $16(1)$ & $9(1)$ & $15(1)$ \\
\hline$C(1)$ & $35(1)$ & $41(1)$ & $43(1)$ & 12 (1) & $10(1)$ & $18(1)$ \\
\hline$C(2)$ & $49(2)$ & $55(2)$ & $59(2)$ & $22(1)$ & $19(1)$ & $15(1)$ \\
\hline$C(3)$ & $90(2)$ & $53(2)$ & $82(2)$ & $24(2)$ & $23(2)$ & $14(2)$ \\
\hline$C(4)$ & $64(2)$ & $35(1)$ & $57(2)$ & $9(1)$ & $6(1)$ & $14(1)$ \\
\hline$C(5)$ & $27(1)$ & $35(1)$ & $44(1)$ & $8(1)$ & $2(1)$ & $12(1)$ \\
\hline$C(6)$ & $34(1)$ & $34(1)$ & $38(1)$ & $5(1)$ & $5(1)$ & $15(1)$ \\
\hline$C(7)$ & $46(2)$ & $74(2)$ & $53(1)$ & 25 (1) & $19(1)$ & $38(1)$ \\
\hline$C(8)$ & $51(1)$ & $40(1)$ & $50(1)$ & $6(1)$ & $14(1)$ & $20(1)$ \\
\hline$C(9)$ & $65(2)$ & $80(2)$ & $68(2)$ & $-16(2)$ & $-19(1)$ & $16(2)$ \\
\hline
\end{tabular}


Compound $(2 R, 7 \mathrm{a} R)-23 \mathrm{a}:$ : Orthorhombic, space group P 2121 21, $a=6.527(1), b=10.719(1)$, $c=23.553(3) \AA, \mathrm{V}=1647.8(15) \AA^{3}, \mathrm{Z}=4 \mathrm{D}_{\mathrm{c}}=1.219, \mu=0.680 \mathrm{~mm}^{-1}, \mathrm{~F}(000)=648$. The reflections collected were 2084 with a $3.75<\theta<64.18$ range; 1897 were independent and the final $\mathrm{R}$ index was 0.0387 for reflections having $\mathrm{I}>2 \sigma \mathrm{I}$, and 0.0389 for all data.

Table s5. Crystal data and structure refinement for (2R,7aR)-23a.

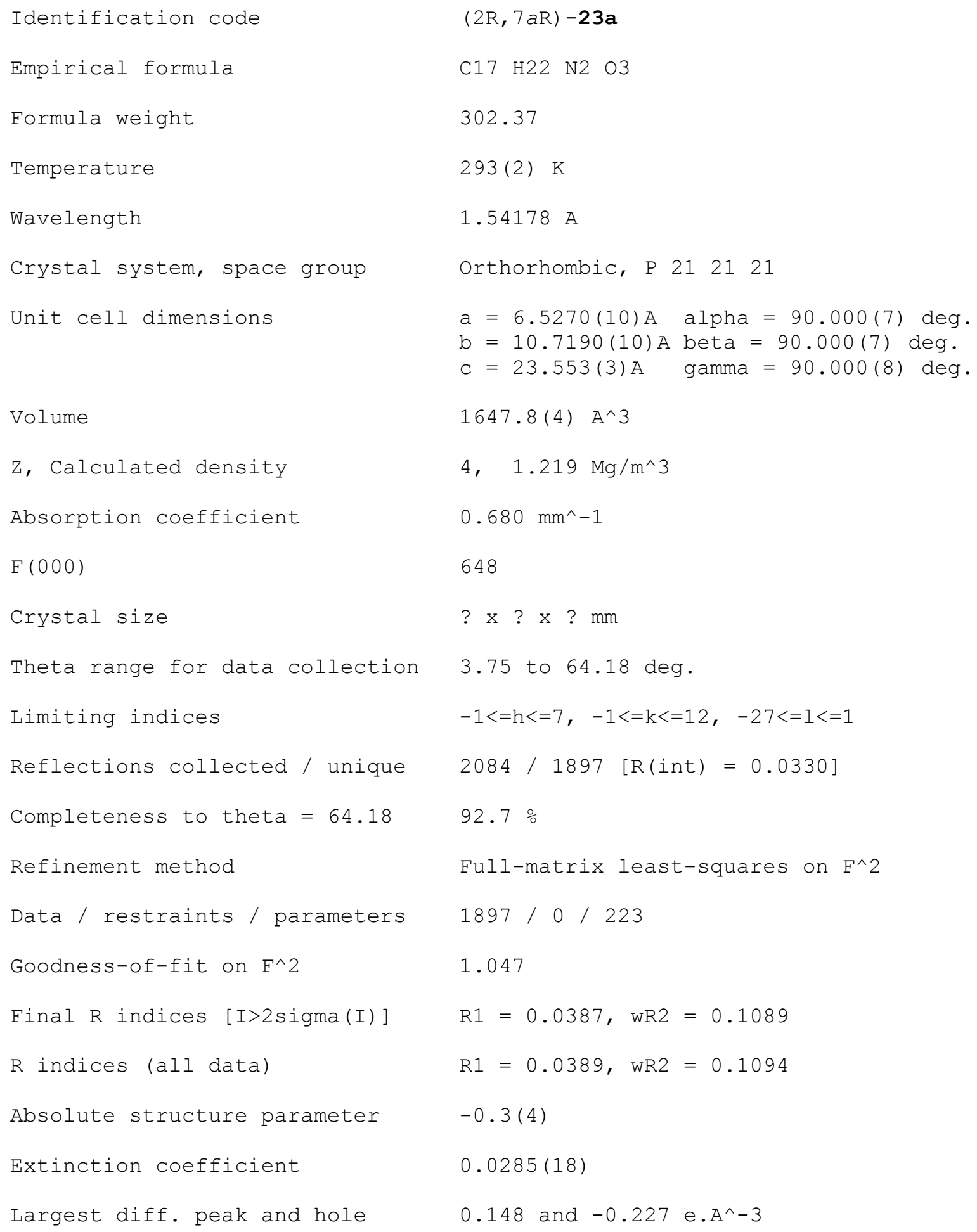


Table s6. Atomic coordinates ( $x$ 10^4) and equivalent isotropic displacement parameters $\left(A^{\wedge} 2 \times 10^{\wedge} 3\right)$ for $(2 R, 7 a R)-23 a$.

$\mathrm{U}(\mathrm{eq})$ is defined as one third of the trace of the orthogonalized Uij tensor.

\begin{tabular}{|c|c|c|c|c|}
\hline & $\mathrm{x}$ & $\mathrm{Y}$ & $\mathrm{z}$ & $\mathrm{U}(\mathrm{eq})$ \\
\hline $\mathrm{N}(1)$ & 1915 (3) & $560(2)$ & $8285(1)$ & $51(1)$ \\
\hline $\mathrm{N}(2)$ & $5586(3)$ & $1579(2)$ & $7334(1)$ & $50(1)$ \\
\hline$O(1)$ & $2795(3)$ & $-653(1)$ & $7529(1)$ & $64(1)$ \\
\hline$O(2)$ & $3253(5)$ & $1334(2)$ & $9338(1)$ & $111(1)$ \\
\hline$O(3)$ & $2279(5)$ & $3275(2)$ & $9180(1)$ & $94(1)$ \\
\hline$C(1)$ & $1754(4)$ & $1881(2)$ & $8432(1)$ & $49(1)$ \\
\hline$C(2)$ & $-568(4)$ & $2098(3)$ & $8398(1)$ & $64(1)$ \\
\hline$C(3)$ & $-1465(4)$ & $879(3)$ & $8614(1)$ & $73(1)$ \\
\hline$C(4)$ & $139(4)$ & $-138(2)$ & $8496(1)$ & $67(1)$ \\
\hline$C(5)$ & $2749(3)$ & $362(2)$ & $7766(1)$ & $49(1)$ \\
\hline$C(6)$ & $3465(3)$ & $1603(2)$ & $7518(1)$ & $47(1)$ \\
\hline$C(7)$ & $3134(5)$ & $2544(2)$ & $7996(1)$ & $58(1)$ \\
\hline$C(8)$ & $2523(4)$ & $2093(2)$ & $9032(1)$ & $59(1)$ \\
\hline$C(9)$ & $2911(7)$ & $3635(3)$ & $9744(1)$ & $109(1)$ \\
\hline$C(10)$ & $5955(3)$ & $990(2)$ & $6778(1)$ & $50(1)$ \\
\hline$C(11)$ & $8247(4)$ & $1004(3)$ & $6662(1)$ & $69(1)$ \\
\hline C (12) & $4761(3)$ & $1567(2)$ & $6291(1)$ & $48(1)$ \\
\hline$C(13)$ & $3517(4)$ & $841(3)$ & $5954(1)$ & $71(1)$ \\
\hline$C(14)$ & $2473(5)$ & $1364(4)$ & $5492(1)$ & $91(1)$ \\
\hline$C(15)$ & $2675(5)$ & $2605(3)$ & $5370(1)$ & $82(1)$ \\
\hline$C(16)$ & $3894(5)$ & $3338(3)$ & $5704(1)$ & $72(1)$ \\
\hline$C(17)$ & $4924(4)$ & $2827(2)$ & $6161(1)$ & $59(1)$ \\
\hline
\end{tabular}

Table s7. Bond lengths $[A]$ and angles [deg] for (2R, $7 a R)-\mathbf{2 3 a}$.

$\begin{array}{ll}\mathrm{N}(1)-\mathrm{C}(5) & 1.356(3) \\ \mathrm{N}(1)-\mathrm{C}(1) & 1.461(2) \\ \mathrm{N}(1)-\mathrm{C}(4) & 1.466(3) \\ \mathrm{N}(2)-\mathrm{C}(6) & 1.451(3) \\ \mathrm{N}(2)-\mathrm{C}(10) & 1.473(3) \\ \mathrm{N}(2)-\mathrm{HN} 2 & 0.85(3) \\ \mathrm{O}(1)-\mathrm{C}(5) & 1.223(2) \\ \mathrm{O}(2)-\mathrm{C}(8) & 1.186(3) \\ \mathrm{O}(3)-\mathrm{C}(8) & 1.323(3) \\ \mathrm{O}(3)-\mathrm{C}(9) & 1.444(3) \\ \mathrm{C}(1)-\mathrm{C}(8) & 1.517(3) \\ \mathrm{C}(1)-\mathrm{C}(2) & 1.535(3) \\ \mathrm{C}(1)-\mathrm{C}(7) & 1.542(3) \\ \mathrm{C}(2)-\mathrm{C}(3) & 1.520(4) \\ \mathrm{C}(2)-\mathrm{H}(2 \mathrm{~A}) & 0.9700 \\ \mathrm{C}(2)-\mathrm{H}(2 \mathrm{~B}) & 0.9700 \\ \mathrm{C}(3)-\mathrm{C}(4) & 1.537(4) \\ \mathrm{C}(3)-\mathrm{H}(3 \mathrm{~A}) & 0.9700 \\ \mathrm{C}(3)-\mathrm{H}(3 \mathrm{~B}) & 0.9700 \\ \mathrm{C}(4)-\mathrm{H}(4 \mathrm{~A}) & 0.9700 \\ \mathrm{C}(4)-\mathrm{H}(4 \mathrm{~B}) & 0.9700 \\ \mathrm{C}(5)-\mathrm{C}(6) & 1.526(3) \\ \mathrm{C}(6)-\mathrm{C}(7) & 1.527(3) \\ \mathrm{C}(6)-\mathrm{H}(6) & 0.96(2) \\ \mathrm{C}(7)-\mathrm{H}(7 \mathrm{~A}) & 0.99(3) \\ \mathrm{C}(7)-\mathrm{H}(7 \mathrm{~B}) & 1.12(3) \\ & \end{array}$




\begin{tabular}{|c|c|}
\hline $\mathrm{C}(9)-\mathrm{H}(9 \mathrm{~A})$ & 0.9600 \\
\hline$C(9)-H(9 B)$ & 0.9600 \\
\hline $\mathrm{C}(9)-\mathrm{H}(9 \mathrm{C})$ & 0.9600 \\
\hline$C(10)-C(12)$ & $1.520(3)$ \\
\hline$C(10)-C(11)$ & $1.521(3)$ \\
\hline $\mathrm{C}(10)-\mathrm{H}(10)$ & $0.98(2)$ \\
\hline $\mathrm{C}(11)-\mathrm{H}(11 \mathrm{~A})$ & 0.9600 \\
\hline$C(11)-H(11 B)$ & 0.9600 \\
\hline $\mathrm{C}(11)-\mathrm{H}(11 \mathrm{C})$ & 0.9600 \\
\hline$C(12)-C(13)$ & $1.377(3)$ \\
\hline$C(12)-C(17)$ & $1.388(3)$ \\
\hline$C(13)-C(14)$ & $1.401(4)$ \\
\hline $\mathrm{C}(13)-\mathrm{H}(13)$ & 0.9300 \\
\hline$C(14)-C(15)$ & $1.368(5)$ \\
\hline $\mathrm{C}(14)-\mathrm{H}(14)$ & 0.9300 \\
\hline$C(15)-C(16)$ & $1.367(4)$ \\
\hline $\mathrm{C}(15)-\mathrm{H}(15)$ & 0.9300 \\
\hline$C(16)-C(17)$ & $1.383(3)$ \\
\hline $\mathrm{C}(16)-\mathrm{H}(16)$ & 0.9300 \\
\hline $\mathrm{C}(17)-\mathrm{H}(17)$ & 0.9300 \\
\hline $\mathrm{C}(5)-\mathrm{N}(1)-\mathrm{C}(1)$ & $113.27(16)$ \\
\hline $\mathrm{C}(5)-\mathrm{N}(1)-\mathrm{C}(4)$ & $122.87(18)$ \\
\hline $\mathrm{C}(1)-\mathrm{N}(1)-\mathrm{C}(4)$ & $110.94(18)$ \\
\hline$C(6)-N(2)-C(10)$ & $115.36(16)$ \\
\hline $\mathrm{C}(6)-\mathrm{N}(2)-\mathrm{HN} 2$ & $109.5(18)$ \\
\hline $\mathrm{C}(10)-\mathrm{N}(2)-\mathrm{HN} 2$ & $107.6(17)$ \\
\hline$C(8)-O(3)-C(9)$ & $117.6(2)$ \\
\hline $\mathrm{N}(1)-\mathrm{C}(1)-\mathrm{C}(8)$ & $110.04(17)$ \\
\hline $\mathrm{N}(1)-\mathrm{C}(1)-\mathrm{C}(2)$ & $101.86(19)$ \\
\hline$C(8)-C(1)-C(2)$ & $110.70(19)$ \\
\hline $\mathrm{N}(1)-\mathrm{C}(1)-\mathrm{C}(7)$ & $104.27(16)$ \\
\hline$C(8)-C(1)-C(7)$ & $111.05(18)$ \\
\hline$C(2)-C(1)-C(7)$ & $118.1(2)$ \\
\hline$C(3)-C(2)-C(1)$ & $103.4(2)$ \\
\hline $\mathrm{C}(3)-\mathrm{C}(2)-\mathrm{H}(2 \mathrm{~A})$ & 111.1 \\
\hline $\mathrm{C}(1)-\mathrm{C}(2)-\mathrm{H}(2 \mathrm{~A})$ & 111.1 \\
\hline$C(3)-C(2)-H(2 B)$ & 111.1 \\
\hline $\mathrm{C}(1)-\mathrm{C}(2)-\mathrm{H}(2 \mathrm{~B})$ & 111.1 \\
\hline $\mathrm{H}(2 \mathrm{~A})-\mathrm{C}(2)-\mathrm{H}(2 \mathrm{~B})$ & 109.0 \\
\hline$C(2)-C(3)-C(4)$ & $106.7(2)$ \\
\hline$C(2)-C(3)-H(3 A)$ & 110.4 \\
\hline $\mathrm{C}(4)-\mathrm{C}(3)-\mathrm{H}(3 \mathrm{~A})$ & 110.4 \\
\hline$C(2)-C(3)-H(3 B)$ & 110.4 \\
\hline$C(4)-C(3)-H(3 B)$ & 110.4 \\
\hline $\mathrm{H}(3 \mathrm{~A})-\mathrm{C}(3)-\mathrm{H}(3 \mathrm{~B})$ & 108.6 \\
\hline$N(1)-C(4)-C(3)$ & $103.75(19)$ \\
\hline $\mathrm{N}(1)-\mathrm{C}(4)-\mathrm{H}(4 \mathrm{~A})$ & 111.0 \\
\hline $\mathrm{C}(3)-\mathrm{C}(4)-\mathrm{H}(4 \mathrm{~A})$ & 111.0 \\
\hline $\mathrm{N}(1)-\mathrm{C}(4)-\mathrm{H}(4 \mathrm{~B})$ & 111.0 \\
\hline$C(3)-C(4)-H(4 B)$ & 111.0 \\
\hline $\mathrm{H}(4 \mathrm{~A})-\mathrm{C}(4)-\mathrm{H}(4 \mathrm{~B})$ & 109.0 \\
\hline $\mathrm{O}(1)-\mathrm{C}(5)-\mathrm{N}(1)$ & $124.12(18)$ \\
\hline $\mathrm{O}(1)-\mathrm{C}(5)-\mathrm{C}(6)$ & $126.40(17)$ \\
\hline$N(1)-C(5)-C(6)$ & $109.36(15)$ \\
\hline$N(2)-C(6)-C(5)$ & $113.02(17)$ \\
\hline$N(2)-C(6)-C(7)$ & $111.49(19)$ \\
\hline$C(5)-C(6)-C(7)$ & $104.51(16)$ \\
\hline $\mathrm{N}(2)-\mathrm{C}(6)-\mathrm{H}(6)$ & $115.0(12)$ \\
\hline $\mathrm{C}(5)-\mathrm{C}(6)-\mathrm{H}(6)$ & $103.1(13)$ \\
\hline $\mathrm{C}(7)-\mathrm{C}(6)-\mathrm{H}(6)$ & $108.9(13)$ \\
\hline$C(6)-C(7)-C(1)$ & $105.66(17)$ \\
\hline$C(6)-C(7)-H(7 A)$ & $109.4(17)$ \\
\hline $\mathrm{C}(1)-\mathrm{C}(7)-\mathrm{H}(7 \mathrm{~A})$ & $112.2(18)$ \\
\hline
\end{tabular}




\begin{tabular}{|c|c|}
\hline$C(6)-C(7)-H(7 B)$ & $107.9(15)$ \\
\hline $\mathrm{C}(1)-\mathrm{C}(7)-\mathrm{H}(7 \mathrm{~B})$ & $109.0(15)$ \\
\hline $\mathrm{H}(7 \mathrm{~A})-\mathrm{C}(7)-\mathrm{H}(7 \mathrm{~B})$ & $112(3)$ \\
\hline $\mathrm{O}(2)-\mathrm{C}(8)-\mathrm{O}(3)$ & $123.1(2)$ \\
\hline $\mathrm{O}(2)-\mathrm{C}(8)-\mathrm{C}(1)$ & $126.6(2)$ \\
\hline $\mathrm{O}(3)-\mathrm{C}(8)-\mathrm{C}(1)$ & $110.4(2)$ \\
\hline $\mathrm{O}(3)-\mathrm{C}(9)-\mathrm{H}(9 \mathrm{~A})$ & 109.5 \\
\hline$O(3)-C(9)-H(9 B)$ & 109.5 \\
\hline $\mathrm{H}(9 \mathrm{~A})-\mathrm{C}(9)-\mathrm{H}(9 \mathrm{~B})$ & 109.5 \\
\hline $\mathrm{O}(3)-\mathrm{C}(9)-\mathrm{H}(9 \mathrm{C})$ & 109.5 \\
\hline $\mathrm{H}(9 \mathrm{~A})-\mathrm{C}(9)-\mathrm{H}(9 \mathrm{C})$ & 109.5 \\
\hline $\mathrm{H}(9 \mathrm{~B})-\mathrm{C}(9)-\mathrm{H}(9 \mathrm{C})$ & 109.5 \\
\hline$N(2)-C(10)-C(12)$ & $114.40(17)$ \\
\hline $\mathrm{N}(2)-\mathrm{C}(10)-\mathrm{C}(11)$ & $108.45(19)$ \\
\hline$C(12)-C(10)-C(11)$ & $111.4(2)$ \\
\hline $\mathrm{N}(2)-\mathrm{C}(10)-\mathrm{H}(10)$ & $107.2(12)$ \\
\hline $\mathrm{C}(12)-\mathrm{C}(10)-\mathrm{H}(10)$ & $105.6(12)$ \\
\hline $\mathrm{C}(11)-\mathrm{C}(10)-\mathrm{H}(10)$ & $109.7(13)$ \\
\hline $\mathrm{C}(10)-\mathrm{C}(11)-\mathrm{H}(11 \mathrm{~A})$ & 109.5 \\
\hline $\mathrm{C}(10)-\mathrm{C}(11)-\mathrm{H}(11 \mathrm{~B})$ & 109.5 \\
\hline $\mathrm{H}(11 \mathrm{~A})-\mathrm{C}(11)-\mathrm{H}(11 \mathrm{~B})$ & 109.5 \\
\hline $\mathrm{C}(10)-\mathrm{C}(11)-\mathrm{H}(11 \mathrm{C})$ & 109.5 \\
\hline $\mathrm{H}(11 \mathrm{~A})-\mathrm{C}(11)-\mathrm{H}(11 \mathrm{C})$ & 109.5 \\
\hline $\mathrm{H}(11 \mathrm{~B})-\mathrm{C}(11)-\mathrm{H}(11 \mathrm{C})$ & 109.5 \\
\hline$C(13)-C(12)-C(17)$ & $118.0(2)$ \\
\hline$C(13)-C(12)-C(10)$ & $120.5(2)$ \\
\hline$C(17)-C(12)-C(10)$ & $121.52(19)$ \\
\hline$C(12)-C(13)-C(14)$ & $120.5(3)$ \\
\hline $\mathrm{C}(12)-\mathrm{C}(13)-\mathrm{H}(13)$ & 119.7 \\
\hline $\mathrm{C}(14)-\mathrm{C}(13)-\mathrm{H}(13)$ & 119.7 \\
\hline$C(15)-C(14)-C(13)$ & $120.4(3)$ \\
\hline $\mathrm{C}(15)-\mathrm{C}(14)-\mathrm{H}(14)$ & 119.8 \\
\hline $\mathrm{C}(13)-\mathrm{C}(14)-\mathrm{H}(14)$ & 119.8 \\
\hline$C(16)-C(15)-C(14)$ & $119.6(3)$ \\
\hline $\mathrm{C}(16)-\mathrm{C}(15)-\mathrm{H}(15)$ & 120.2 \\
\hline $\mathrm{C}(14)-\mathrm{C}(15)-\mathrm{H}(15)$ & 120.2 \\
\hline$C(15)-C(16)-C(17)$ & $120.3(3)$ \\
\hline $\mathrm{C}(15)-\mathrm{C}(16)-\mathrm{H}(16)$ & 119.9 \\
\hline $\mathrm{C}(17)-\mathrm{C}(16)-\mathrm{H}(16)$ & 119.9 \\
\hline$C(16)-C(17)-C(12)$ & $121.2(2)$ \\
\hline $\mathrm{C}(16)-\mathrm{C}(17)-\mathrm{H}(17)$ & 119.4 \\
\hline $\mathrm{C}(12)-\mathrm{C}(17)-\mathrm{H}(17)$ & 119.4 \\
\hline
\end{tabular}


Table s8. Anisotropic displacement parameters $\left(A^{\wedge} 2 \times 10^{\wedge} 3\right)$ for $(2 R, 7 a R)-$ $23 a$.

The anisotropic displacement factor exponent takes the form:

$-2 \mathrm{pi}^{\wedge} 2\left[\mathrm{~h}^{\wedge} 2 \mathrm{a}^{\star \wedge} 2 \mathrm{U11}+\ldots+2 \mathrm{~h} \mathrm{k}\right.$ a* b* U12 ]

\begin{tabular}{|c|c|c|c|c|c|c|}
\hline & U11 & U22 & U33 & U23 & U13 & $\mathrm{U} 12$ \\
\hline $\mathrm{N}(1)$ & $63(1)$ & $38(1)$ & $52(1)$ & $3(1)$ & $5(1)$ & $-1(1)$ \\
\hline$N(2)$ & $53(1)$ & $44(1)$ & $52(1)$ & $2(1)$ & $-8(1)$ & $-5(1)$ \\
\hline$O(1)$ & $81(1)$ & $40(1)$ & $70(1)$ & $-7(1)$ & $9(1)$ & $-3(1)$ \\
\hline$O(2)$ & $167(2)$ & $89(1)$ & $77(1)$ & $6(1)$ & $-50(2)$ & $19(2)$ \\
\hline$O(3)$ & $149(2)$ & $70(1)$ & $61(1)$ & $-23(1)$ & $-21(1)$ & $12(1)$ \\
\hline$C(1)$ & $62(1)$ & $40(1)$ & $46(1)$ & $-4(1)$ & $-3(1)$ & $3(1)$ \\
\hline$C(2)$ & $62(1)$ & $62(1)$ & $68(1)$ & $-3(1)$ & $-9(1)$ & $11(1)$ \\
\hline$C(3)$ & $62(1)$ & $77(2)$ & $79(1)$ & $-2(1)$ & $6(1)$ & $0(1)$ \\
\hline$C(4)$ & $69(1)$ & $58(1)$ & $74(1)$ & $4(1)$ & $13(1)$ & $-8(1)$ \\
\hline$C(5)$ & $55(1)$ & $40(1)$ & $53(1)$ & $-1(1)$ & $-2(1)$ & $2(1)$ \\
\hline$C(6)$ & $57(1)$ & $41(1)$ & $43(1)$ & $-1(1)$ & $-4(1)$ & $1(1)$ \\
\hline$C(7)$ & $81(2)$ & $38(1)$ & $55(1)$ & $-3(1)$ & $4(1)$ & $-1(1)$ \\
\hline$C(8)$ & $65(1)$ & $62(1)$ & $51(1)$ & $-1(1)$ & $-4(1)$ & $4(1)$ \\
\hline C (9) & $150(3)$ & $114(2)$ & $62(1)$ & $-33(2)$ & $-6(2)$ & $-19(3)$ \\
\hline$C(10)$ & $49(1)$ & $43(1)$ & $58(1)$ & $1(1)$ & $0(1)$ & $1(1)$ \\
\hline$C(11)$ & $50(1)$ & $69(1)$ & $88(2)$ & $-4(1)$ & $1(1)$ & $6(1)$ \\
\hline$C(12)$ & $45(1)$ & $53(1)$ & $47(1)$ & $-5(1)$ & $6(1)$ & $0(1)$ \\
\hline$C(13)$ & $69(1)$ & $74(2)$ & 71 (1) & 1 (1) & $-11(1)$ & $-18(1)$ \\
\hline$C(14)$ & $81(2)$ & $113(2)$ & $78(2)$ & $3(2)$ & $-26(2)$ & $-25(2)$ \\
\hline$C(15)$ & $72(2)$ & $112(2)$ & $63(1)$ & $16(2)$ & $-8(1)$ & $5(2)$ \\
\hline$C(16)$ & $91(2)$ & $69(1)$ & $55(1)$ & 7 (1) & $9(1)$ & $16(2)$ \\
\hline$C(17)$ & $75(1)$ & $55(1)$ & $49(1)$ & $-4(1)$ & $0(1)$ & $5(1)$ \\
\hline
\end{tabular}

\section{References:}

S1. Walker, N.; Stuart, D. Acta Cystallogr. Sect. A, 1983, 39, 158-166.

S2. Altomare, A.; Burla, M. C.; Cavalli, M.; Cascarano, G. L., Giacovazzo; C., Gagliardi; A., Moliterni, A. G. G.; Polidori, G.; Spagna, R. J. Appl. Cryst., 1999, 32, 115-119.

S3. Sheldrick, G, M. SHELXL97: Program for Crystal Structure Refinement; Institut für Anorganische Chemie de Universitat Göttingen. Göttingen, Germany. 